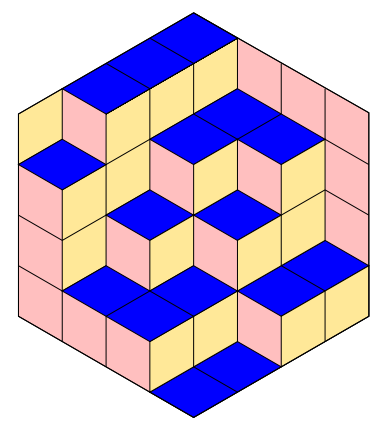

Clara Franchi, Alexander A. Ivanov \& Mario Mainardis Radicals of $S_{n}$-invariant positive semidefinite hermitian forms Volume 1, issue 4 (2018), p. 425-440.

<http://alco.centre-mersenne.org/item/ALCO_2018__1_4_425_0>

(c) The journal and the authors, 2018.

Some rights reserved.

\begin{tabular}{|l|l}
\hline (c) BY This article is licensed under the \\
\hline
\end{tabular}

Creative Commons ATtribution 4.0 InTERnational License.

http://creativecommons.org/licenses/by/4.0/

Access to articles published by the journal Algebraic Combinatorics on the website http://alco.centre-mersenne.org/ implies agreement with the Terms of Use (http://alco.centre-mersenne.org/legal/).

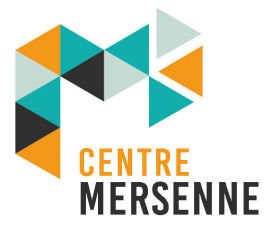

Algebraic Combinatorics is member of the Centre Mersenne for Open Scientific Publishing www.centre-mersenne.org 


\title{
Radicals of $S_{n}$-invariant positive semidefinite hermitian forms
}

\author{
Clara Franchi, Alexander A. Ivanov \& Mario Mainardis
}

\begin{abstract}
Let $G$ be a finite group, $V$ a complex permutation module for $G$ over a finite $G$-set $\mathcal{X}$, and $f: V \times V \rightarrow \mathbb{C}$ a $G$-invariant positive semidefinite hermitian form on $V$. In this paper we show how to compute the radical $V^{\perp}$ of $f$, by extending to nontransitive actions the classical combinatorial methods from the theory of association schemes. We apply this machinery to obtain a result for standard Majorana representations of the symmetric groups.
\end{abstract}

\section{INTRODUCTION}

A major difficulty in studying linear representations of certain finite groups, such as the large sporadic simple groups, arises when the degrees of these representations become so large that applying the general methods from linear algebra gets hard, if not practically impossible, even by machine computation. In this paper we cope with a frequent problem when dealing with the usual representation of the Monster (and many if its simple subgroups) on the Norton-Conway-Griess algebra, or, more generally, with Majorana representations of finite groups (see [7]), and can be stated as follows: given a finite group $G$, a complex permutation module $V$ on a finite $G$-set $\mathcal{X}$, and a $G$-invariant positive semidefinite hermitian form $f$, determine the radical $V^{\perp}$ of $f$ from the Gram matrix $\Gamma$ associated to $f$ with respect to $\mathcal{X}$. In this context, the $G$-invariance of the form $f$ implies strong restrictions on the Gram matrix $\Gamma$ that can be exploited, via the theory of association schemes, to get a significantly more manageable situation. In fact, by [5, p. 11] or $[15, \S 2.6$ and $\S 2.7], \Gamma$ is equivalent to a block diagonal matrix $\Gamma^{\prime}$, whose blocks have sizes corresponding to the multiplicities of the irreducible $\mathbb{C}[G]$-submodules of $V$, so that the decomposition of $V^{\perp}$ into irreducible $\mathbb{C}[G]$-submodules can be recovered from the ranks of the diagonal blocks of $\Gamma^{\prime}$. The key step to compute the diagonal blocks of $\Gamma^{\prime}$ is to determine a generalised first eigenmatrix (see [3]) of the association scheme related to the action of $G$ on $\mathcal{X}$. If this action is multiplicity-free (or, better, if the graph associated to this action is distance transitive), there are well established combinatorial methods (see [1] or [4]) to compute this matrix. On the other hand, if the action is not multiplicity-free, this strategy becomes much more awkward, though still possible in some cases: in [3], for example, this machinery has been extended to the case where at most one irreducible $\mathbb{C}[G]$-submodule of the complex permutation module on $\mathcal{X}$ has multiplicity 2 and all the others have multiplicity 1 (a more detailed description on how to deal with this

Manuscript received 3rd August 2017, revised 4th June 2018, accepted 10th June 2018.

Keywords. Hermitian form, Symmetric group, Majorana representation, Monster group, Association scheme, Specht module. 


\begin{tabular}{|c|c|c|}
\hline$(x, y)^{S_{n}}$ & $f(x, y)$ & \\
\hline$((1,2)(3,4),(1,2)(3,4))^{S_{n}}$ & 1 & $n \geqslant 4$ \\
\hline$((1,2)(3,4),(1,3)(2,4))^{S_{n}}$ & $1 / 8$ & $n \geqslant 4$ \\
\hline$((1,2)(3,4),(1,2)(3,5))^{S_{n}}$ & $13 / 2^{8}$ & $n \geqslant 5$ \\
\hline$((1,2)(3,4),(1,3)(2,5))^{S_{n}}$ & $3 / 2^{7}$ & $n \geqslant 5$ \\
\hline$((1,2)(3,4),(1,2)(5,6))^{S_{n}}$ & $1 / 8$ & $n \geqslant 6$ \\
\hline$((1,2)(3,4),(1,3)(5,6))^{S_{n}}$ & $1 / 2^{6}$ & $n \geqslant 6$ \\
\hline$((1,2)(3,4),(1,5)(2,6))^{S_{n}}$ & $1 / 2^{6}$ & $n \geqslant 6$ \\
\hline$((1,2)(3,4),(1,5)(3,6))^{S_{n}}$ & $13 / 2^{8}$ & $n \geqslant 6$ \\
\hline$((1,2)(3,4),(1,5)(6,7))^{S_{n}}$ & $5 / 2^{8}$ & $n \geqslant 7$ \\
\hline$((1,2)(3,4),(5,6)(7,8))^{S_{n}}$ & 0 & $n \geqslant 8$ \\
\hline$(\{1,2,3\},\{1,2,3\})^{S_{n}}$ & $2^{3} / 5$ & $n \geqslant 3$ \\
\hline$(\{1,2,3\},\{1,2,4\})^{S_{n}}$ & $2^{3} \cdot 17 /\left(3^{4} \cdot 5\right)$ & $n \geqslant 4$ \\
\hline$(\{1,2,3\},\{1,4,5\})^{S_{n}}$ & $2^{4} /\left(3^{4} \cdot 5\right)$ & $n \geqslant 5$ \\
\hline$(\{1,2,3\},\{4,5,6\})^{S_{n}}$ & 0 & $n \geqslant 6$ \\
\hline$(\{1,2,3\},(1,2)(3,4))^{S_{n}}$ & $1 / 3^{2}$ & $n \geqslant 4$ \\
\hline$(\{1,2,5\},(1,2)(3,4))^{S_{n}}$ & $1 / 4$ & $n \geqslant 5$ \\
\hline$(\{1,3,5\},(1,2)(3,4))^{S_{n}}$ & $1 /\left(2 \cdot 3^{2}\right)$ & $n \geqslant 5$ \\
\hline$(\{1,5,6\},(1,2)(3,4))^{S_{n}}$ & $1 /\left(2^{2} \cdot 3^{2}\right)$ & $n \geqslant 6$ \\
\hline$(\{5,6,7\},(1,2)(3,4))^{S_{n}}$ & 0 & $n \geqslant 7$ \\
\hline
\end{tabular}

TABLE 1. The relevant values of the form $f$

case will be given in Section 3). We'll show in the sequel how, in the case of nontransitive actions (which are definitely not multiplicity-free), $V^{\perp}$ can be determined from the generalised first eigenmatrices of the association schemes related to the actions induced by $G$ on the $G$-orbits of $\mathcal{X}$. As an application, we prove the following result:

THEOREM 1.1. Let $n$ be a positive integer with $4 \leqslant n, S_{n}$ the symmetric group on $\{1, \ldots, n\}, \mathcal{T}$ the set of the permutations of $S_{n}$ of type $(2,2)$, on which $S_{n}$ acts via conjugation, $\mathcal{U}$ the set of 3 -subsets of $\{1, \ldots, n\}$, on which $S_{n}$ acts in the natural way, $\mathcal{X}$ the union (as $S_{n}$-sets) of $\mathcal{T}$ with $\mathcal{U}, V$ the complex permutation module of $S_{n}$ on $\mathcal{X}$, and $f$ the $S_{n}$-invariant hermitian form on $V$ defined as in Table 1. Then, denoting by $S^{\lambda}$ the Specht module associated to a partition $\lambda$ of $\{1, \ldots, n\}$, we have

(1) $f$ is positive semidefinite if and only if $n \leqslant 12$;

(2) if $n=12$, then $V^{\perp} \cong 1 \oplus S^{(11,1)} \oplus 2 S^{(10,2)} \oplus S^{(9,3)} \oplus S^{(9,2,1)}$;

(3) if $n=11$, then $V^{\perp} \cong S^{(2,9)}$;

(4) if $10 \geqslant n \geqslant 8$, then $V^{\perp}=\{0\}$.

We remark that under the hypothesis of Theorem 1.1, $S^{(n-2,2)}$ has multiplicity 3 in $V$ and the homogeneous component $V\left(S^{(n-2,2)}\right)$ splits into the orthogonal direct sum of its intersections with the linear spans $T$ and $U$ of $\mathcal{T}$ and $\mathcal{U}$, respectively. In $U$ the module $S^{(n-2,2)}$ has multiplicity 1 and, as we shall see, $V\left(S^{(n-2,2)}\right) \cap T$ splits as the direct sum of an irreducible submodule, canonically associated to the Johnson scheme $J(n, 4)$, and its orthogonal complement with respect to a natural $S_{n^{-}}$ invariant hermitian form $\kappa$ defined in Section 3. The choices of $\mathcal{X}$ and $f$ in Theorem 1.1 arise from the theory of Majorana representations; in particular, the form $f$ is the one induced on $V$ by a standard Majorana representation of $S_{n}$ and Theorem 1.1 is 
needed to determine the subalgebra generated by the Majorana axes associated to this representation. When $n<8$, this subalgebra has been determined by Ivanov, Seress, Pasechnik, and Shpectorov in [8], [9], [10]. Since the Specht modules are defined over $\mathbb{Q}$ and they are absolutely irreducible [11, Theorem 4.12], restricting the scalars to $\mathbb{R}$, we obtain immediately from Theorem 1.1 the following result about Majorana representations of the symmetric groups:

THEOREM 1.2. Let $n$ be an integer greater than 7 and $S_{n}$ the symmetric group on $\{1, \ldots, n\}$. Let $\left(S_{n}, \mathcal{T}, W, \phi, \psi\right)$ be a standard Majorana representation of $S_{n}, Y$ and $Z$ be the subspaces of $W$ generated by the axial vectors associated to the bitranspositions and the 3-cycles of $S_{n}$ respectively. Then

(1) $n \leqslant 12$;

(2) if $n=12$, then $Z \leqslant Y$, and $Y$ decomposes into irreducible $\mathbb{R}\left[S_{n}\right]$-submodules as follows

$$
1 \oplus S^{(11,1)} \oplus S^{(10,2)} \oplus S^{(9,3)} \oplus S^{(8,4)} \oplus S^{(8,2,2)}
$$

(3) if $n=11$, then $Y \cap Z \cong S^{(9,2)}$, and $Y+Z$ decomposes into irreducible $\mathbb{R}\left[S_{n}\right]$ submodules as follows

$$
1 \oplus 1 \oplus 2 S^{(10,1)} \oplus 2 S^{(9,2)} \oplus 2 S^{(8,3)} \oplus S^{(7,4)} \oplus S^{(8,2,1)} \oplus S^{(7,2,2)} ;
$$

(4) if $n \in\{8,9,10\}, Y \cap Z=\{0\}$ and $Y+Z$ decomposes into irreducible $\mathbb{R}\left[S_{n}\right]$ submodules as follows

$$
1 \oplus 1 \oplus 2 S^{(n-1,1)} \oplus 3 S^{(n-2,2)} \oplus 2 S^{(n-3,3)} \oplus S^{(n-4,4)} \oplus S^{(n-3,2,1)} \oplus S^{(n-4,2,2)} .
$$

Note that, for $n=12$, the inclusion $Z \leqslant Y$ in Theorem 1.2 was proved, with different methos, by Castillo-Ramirez and Ivanov in [2].

\section{Strategy}

Let $G$ be a finite group acting on a finite set $\mathcal{X}:=\left\{x_{1}, \ldots, x_{m}\right\}, V$ the complex permutation module of $G$ on $\mathcal{X}$, and $f$ a $G$-invariant hermitian form on $V$. Let $\Gamma$ be the Gram matrix associated to $f$ with respect to the basis $\left(x_{1}, \ldots, x_{m}\right)$. As stated in the introduction, for each irreducible $\mathbb{C}[G]$-submodule of $V$, we want to determine its multiplicity in $V^{\perp}$ in terms of the matrix $\Gamma$. For the remainder of this paper all modules are $\mathbb{C}[G]$-modules. For an irreducible submodule $S$ of a module $N$, denote by $N(S)$ the $S$-homogeneous component of $N$ (i.e. the submodule of $N$ generated by all submodules of $N$ isomorphic to $S$ ) and by $m_{N}(S)$ the multiplicity of $S$ in $N$. By Maschke's Theorem, $V$ is a completely reducible module, so that $V$ is the direct sum of its homogeneous components.

LEMMA 2.1. Each two distinct homogeneous components of $V$ are orthogonal to each other.

Proof. Since $f$ is positive semidefinite and $G$-invariant, each submodule of $V$ has a $G$-invariant orthogonal complement. So $V$ decomposes as an orthogonal direct sum of irreducible submodules and the result follows by Schur's Lemma.

COROLlary 2.2. Let $S$ be an irreducible submodule of $V$ and assume $V(S)$ is contained in the linear span $W$ of a $G$-orbit $\mathcal{O}$ of $G$ in $\mathcal{X}$. Then the multiplicity of $S$ in $V^{\perp}$ is equal to the multiplicity of $S$ in $W \cap W^{\perp}$.

Proof. By Lemma 2.1, $V(S)$ is orthogonal to the linear span of every $G$-orbit different from $\mathcal{O}$, whence $V(S) \cap V^{\perp}=V(S) \cap W^{\perp}$. 
LEMMA 2.3. Let $N$ and $S$ be finite dimensional modules with $S$ irreducible. Then, for every subgroup $H$ of $G$, we have

$$
\operatorname{dim}_{\mathbb{C}}\left(C_{N(S)}(H)\right)=m_{N}(S) \cdot \operatorname{dim}_{\mathbb{C}}\left(C_{S}(H)\right)
$$

(where, as usual, $C_{N(S)}(H)$ denotes the centraliser of $H$ in $N(S)$, i.e. the set of the vectors in $N(S)$ fixed by $H)$.

Proof. This is an immediate consequence of the complete reducibility of the involved modules.

Corollary 2.4. Assume $f$ is positive semidefinite. Let $S$ be an irreducible $\mathbb{C}[G]$ submodule of $V$, let $H$ be a subgroup of $G$, let $C$ be the centraliser of $H$ in $V(S)$, and let $\Gamma_{(S, H)}$ be the Gram matrix associated to $\left.f\right|_{C \times C}$ with respect to a basis of $C$. Then,

$$
m_{V^{\perp}}(S) \cdot \operatorname{dim}_{\mathbb{C}}\left(C_{S}(H)\right)=\operatorname{corank}\left(\Gamma_{(S, H)}\right) .
$$

Proof. The corank of $\Gamma_{(S, H)}$ is equal to the dimension of $\left(C \cap C^{\perp}\right)$ and, since $f$ is positive semidefinite, $\left(C \cap V^{\perp}\right)=\left(C \cap C^{\perp}\right)$, so the result follows from Lemma 2.3, with $N=V^{\perp}$.

The idea is now to choose $H$ in such a way that $\operatorname{dim}_{\mathbb{C}}\left(C_{S}(H)\right)$ is as small as possible. In particular, if, as in the case we are interested in, $\operatorname{dim}_{\mathbb{C}}\left(C_{S}(H)\right)=1$ then, for every decomposition

$$
V(S)=\bigoplus_{i=1}^{m_{V}(S)} S_{i}
$$

into irreducible submodules $S_{i}$, each one isomorphic with $S$, we can get a basis

$$
\mathcal{C}_{S}:=\left(s_{1}, \ldots, s_{m_{V}(S)}\right)
$$

for $C$ by choosing, for every $i \in\left\{1, \ldots, m_{V}(S)\right\}$, a nontrivial vector $s_{i}$ in $C_{S_{i}}(H)$. A way to obtain the vectors $s_{i}$ is to take the images $t_{i}^{\pi_{i}}$ of suitable $H$-invariant vectors $t_{i}$ of $V$ via the projection $\pi_{i}$ of $V$ onto $S_{i}$ associated to a decomposition of $V$ into irreducible submodules that involves $S_{i}$. The expression of the $s_{i}$ 's as linear combinations of the vectors in $\mathcal{X}$, can be obtained from any generalised first eigenmatrix of the association scheme related to the action of $G$ on $\mathcal{X}$ (see $[5, \S 3]$ ). As already mentioned, if the action of $G$ on $\mathcal{X}$ is not multiplicity-free, there are no standard methods to compute a generalised first eigenmatrix. If the action is not transitive, one might hope to get to a simpler situation by restricting the action to each orbit and decomposing $V(S)$ into the direct sum of its intersections with the linear spans of the $G$-orbits in $\mathcal{X}$. Let

$$
\mathcal{X}_{1}, \ldots, \mathcal{X}_{r}
$$

be the distinct $G$-orbits of $\mathcal{X}$ and let $R_{1}, \ldots, R_{p}$ be representatives of the isomorphism classes of the irreducible submodules of $V$. For $j \in\{1, \ldots, r\}$ let $V_{j}$ be the linear span of $\mathcal{X}_{j}$ and, for $S \in\left\{R_{1}, \ldots, R_{p}\right\}$, let $V_{j}(S):=V(S) \cap V_{j}$, so that $V$ decomposes as follows:

$$
\begin{array}{cccc}
V=V_{1}\left(R_{1}\right) \oplus V_{2}\left(R_{1}\right) \oplus & \cdots & \oplus V_{r}\left(R_{1}\right) \oplus \\
V_{1}\left(R_{2}\right) \oplus V_{2}\left(R_{2}\right) \oplus \cdots & \cdots & V_{r}\left(R_{2}\right) \oplus \\
\vdots & \vdots & \ddots & \vdots \\
V_{1}\left(R_{p}\right) \oplus V_{2}\left(R_{p}\right) \oplus & \cdots & \oplus V_{r}\left(R_{p}\right)
\end{array}
$$

Clearly, for $h \in\{1, \ldots, p\}$, the row sums are the homogeneous components $V\left(R_{h}\right)$ and, for $j \in\{1, \ldots, r\}$, the column sums are the submodules $V_{j}$ 's. For $j \in\{1, \ldots, r\}$ let $\mathcal{O}_{j 1}, \ldots, \mathcal{O}_{j s_{j}}$ be the orbitals of $G$ on $\mathcal{X}_{j}$ and let $P^{\mathcal{X}_{j}}$ be a generalised first eigenmatrix associated to the action of $G$ on $\mathcal{X}_{j}$ (see $[3, \S 2]$ ). Recall that the rows (resp. the columns) of $P^{\mathcal{X}_{j}}$ are in one to one correspondence with isomorphism classes of the 
irreducible submodules of $V_{j}$ (resp. the orbitals of $G$ on $\mathcal{X}_{j}$ ) and the entries of $P^{\mathcal{X}_{j}}$ are square matrices. If $P_{h k}^{j}$ is the $h k$-entry of $P^{\mathcal{X}_{j}}$ corresponding to the irreducible submodule $R_{k}$ and the orbital $\mathcal{O}_{j h}$, the $i l$-entry of $P_{h k}^{j}$ will be denoted by $\left(P_{h k}^{j}\right)_{i l}$. For each such entry define

$$
q_{k h}^{i l}\left(\mathcal{X}_{j}\right):=\frac{\left(\overline{P_{h k}^{j}}\right)_{i l}}{\left|\mathcal{O}_{j h}\right|} \operatorname{dim}_{\mathbb{C}}\left(R_{k}\right)
$$

where $\overline{P_{h k}^{j}}$ is the complex conjugate of the matrix $P_{h k}^{j}$. Let $Q_{k h}^{j}$ be the matrix whose $i l$-entry is $q_{k h}^{i l}\left(\mathcal{X}_{j}\right)$ and let $Q^{\mathcal{X}_{j}}$ be the matrix whose $k h$-entry is $Q_{k h}^{j}$. We shall call $Q^{\mathcal{X}_{j}}$ a generalised second eigenmatrix associated to the action of $G$ on $\mathcal{X}_{j}$.

Lemma 2.5. With the above notation, there exists a decomposition of $V_{j}\left(R_{k}\right)$ as a direct sum of irreducible submodules $S_{1}, \ldots, S_{l}$ (isomorphic to $R_{k}$ ) such that, for every $x \in \mathcal{X}_{j}$, the projection map $\pi_{i}: V \rightarrow S_{i}$ maps

$$
x \mapsto \sum_{h=1}^{s_{j}} q_{k h}^{i i}\left(\mathcal{X}_{j}\right) \sum_{y \in \Delta_{j h}(x)} y,
$$

where $\Delta_{j h}(x):=\left\{y \in \mathcal{X}_{j} \mid(x, y) \in \mathcal{O}_{j h}\right\}$.

Proof. Since the action of $G$ on $\mathcal{X}_{j}$ is transitive, the result follows from [3, Equation (13) and Lemma 1(i)].

Note that in case of a multiplicity-free action $P^{\mathcal{X}_{j}}$ and $Q^{\mathcal{X}_{j}}$ are, respectively, the usual first and second eigenmatrices (see $[1$, p. 60]) and are uniquely determined for fixed orders of the orbitals and of the isomorphism classes of the irreducible submodules. On the other hand, if the action is not multiplicity free, the generalised first eigenmatrix $P^{\mathcal{X}_{j}}$ depends on the chosen decomposition into irreducibles of $V_{j}\left(R_{i}\right)$, for each $i \in\{1, \ldots, p\}$.

\section{TRANSITIVE NON MULTIPLICITY-FREE ACTIONS}

In this section, we briefly describe how the relevant information on a generalised first eigenmatrix $P^{\mathcal{X}_{j}}$ (i.e. the diagonal entries $\left(P_{h k}^{j}\right)_{i i}$ of each block $P_{h k}^{j}$ ) can be obtained in the case where $G$ acts transitively on $\mathcal{X}_{j}$ and all irreducible submodules of the linear span $V_{j}$ of $\mathcal{X}_{j}$ have multiplicity less or equal 2 and only one, which we can assume to be $R_{p}$, has multiplicity 2 . The entries of $P$ relative to an irreducible submodule of multiplicity 1 can be computed inside the submodule itself using Lemma 3 in [3]. To compute the diagonal entries of the $2 \times 2$ blocks corresponding to $R_{p}$, we proceed as follows. Let

$$
\kappa: V_{j} \times V_{j} \rightarrow \mathbb{C}
$$

be the unique nondegenerate hermitian form on $V_{j}$ such that the elements of $\mathcal{X}_{j}$ are mutually orthogonal vectors of norm 1 . Find a $G$-set $\mathcal{Y}$ and a surjective homomorphism of $G$-sets

$$
\theta: \mathcal{X}_{j} \rightarrow \mathcal{Y}
$$

such that, if $M$ is the complex permutation module for $G$ on $\mathcal{Y}$ and $\bar{\theta}: V_{j} \rightarrow M$ is the $\mathbb{C}[G]$-homomorphism induced by $\theta$, the following conditions hold:

(1) $M$ is multiplicity-free;

(2) the first eigenmatrix associated to the action of $G$ on $\mathcal{Y}$ is known;

(3) $M$ contains a submodule isomorphic to $R_{p}$ 
(e.g. in [3], as in Section 4 of this paper, $\mathcal{X}_{j}$ is the set $\mathcal{T}, \mathcal{Y}$ is the set of 4 -subsets of $\{1, \ldots, n\}, \theta$ is the map that sends a permutation of type $(2,2)$ to its support, and $M$ is the Young module $M^{(n-4,4)}$, corresponding to the Johnson scheme $J(n, 4)$ ). Let $I$ be the orthogonal complement to $\operatorname{ker}(\bar{\theta})$ in $V_{j}$ with respect to the form $\kappa$. Since $V_{j}^{\theta}$ contains a submodule isomorphic to $R_{p}$, and $M$ is multiplicity-free, both $I$ and $\operatorname{ker}(\theta)$ contain a copy of $R_{p}$. Denote by $R_{I}$ and $R_{K}$ the copies of $R_{p}$ contained in $I$ and $\operatorname{ker}(\theta)$ respectively. Then $V_{j}\left(R_{p}\right)$ decomposes as the orthogonal (with respect to $\kappa$ ) direct sum of the submodules $R_{I}$ and $R_{K}$ and we get one of the diagonal entries (which we may assume to be the $(1,1)$ entry), given by the following formula (which is an obvious generalisation of Lemma 7 in [3] and is proved in the same way):

$$
\left(P_{k p}^{j}\right)_{11}=c_{l p} \frac{\left|\mathcal{O}_{j k}\right||\mathcal{Y}|}{\left|\overline{\mathcal{O}_{l}}\right|\left|\mathcal{X}_{j}\right|}
$$

where $\overline{\mathcal{O}}_{l}$ is the orbital of $G$ on $\mathcal{Y}$ containing $\mathcal{O}_{j k}^{\theta}$ and $c_{l p}$ is the entry of the first eigenmatrix of $G$ on $\mathcal{Y}$ corresponding to the irreducible module $R_{p}$ and to the orbital $\overline{\mathcal{O}}_{l}$. Finally, for each column of $P$, the last missing "diagonal" entry $\left(P_{k p}^{j}\right)_{22}$ can be computed using the Second Generalised Orthogonality Relation [3, Lemma 1(iii)].

\section{Proof of Theorem 1.1}

From now on let $n, G, \mathcal{T}, \mathcal{U}, \mathcal{X}, V$, and $f$ be as in Theorem 1.1. Let $T$ be the linear span of $\mathcal{T}$ and $U$ the linear span of $\mathcal{U}$, so that $V=T \oplus U$.

Lemma 4.1. With the above notation, $f$ is positive semidefinite if and only if $n \leqslant 12$.

Proof. By [3, Table 13], $T$ has an irreducible submodule isomorphic with $S^{(n-3,2,2)}$ that contains a nonzero vector $v$ such that

$$
f(v, v)=\frac{15}{128}(12-n),
$$

which is clearly negative for $n>12$. Conversely, if $n \leqslant 12$, by [13], we may assume $S_{n}$ to be a subgroup of the Monster such that the bitranspositions of $S_{n}$ are involutions of type $2 A$ in the Monster. Moreover, by [14], there is an $S_{n}$-invariant subset $\mathcal{Y}$ of the (complex) Conway-Norton-Griess algebra $\mathcal{G}$, such that $\mathcal{Y}$ is $S_{n}$-isomorphic to $\mathcal{X}$ and the Gram matrix of the hermitian form $(\cdot, \cdot)_{\mathcal{G}}$ of $\mathcal{G}$ with respect to $\mathcal{Y}$ is the same as $\Gamma$. Since $(\cdot, \cdot)_{\mathcal{G}}$ is positive definite, it follows that $f$ is positive semidefinite on $V$.

4.1. The aCtions of $S_{n}$ ON $\mathcal{T}$ and $\mathcal{U}$. Denote the 10 orbitals

$$
\mathcal{O}_{1}^{\mathcal{T}}, \ldots, \mathcal{O}_{10}^{\mathcal{T}}
$$

of $S_{n}$ on $\mathcal{T}$ and the 4 orbitals

$$
\mathcal{O}_{1}^{\mathcal{U}}, \ldots, \mathcal{O}_{4}^{\mathcal{U}}
$$

of $S_{n}$ on $\mathcal{U}$ as in Table 2. For $\mathcal{R} \in\{\mathcal{T}, \mathcal{U}\}$ and $x \in \mathcal{X} \cap \mathcal{R}$, let

$$
\Delta_{k}^{\mathcal{R}}(x):=\left\{y \in \mathcal{X} \cap \mathcal{R} \mid(x, y) \in \mathcal{O}_{k}^{\mathcal{R}}\right\},
$$

where $k$ ranges from 1 to 10 , if $\mathcal{R}=\mathcal{T}$, and from 1 to 4 , if $\mathcal{R}=\mathcal{U}$.

LEMMA 4.2. Let $T$ be as above, then

(1) T decomposes into irreducible submodules as follows:

$$
T=T_{1,1} \oplus T_{2,1} \oplus T_{3,1} \oplus T_{4,1} \oplus T_{4,2} \oplus T_{5,1} \oplus T_{6,1} \oplus T_{7,1}
$$

where $T_{1,1}$ is the trivial module, $T_{2,1} \cong S^{(n-1,1)}, T_{3,1} \cong S^{(n-3,3)}, T_{4,1} \cong$ $T_{4,2} \cong S^{(n-2,2)}, T_{5,1} \cong S^{(n-4,4)}, T_{6,1} \cong S^{(n-3,2,1)}$, and $T_{7,1} \cong S^{(n-4,2,2)}$. 


$\left|\begin{array}{l}\mathcal{O}_{1}^{\mathcal{T}}:=((1,2)(3,4),(1,2)(3,4))^{S_{n}} \\ \mathcal{O}_{2}^{\mathcal{T}}:=((1,2)(3,4),(1,3)(2,4))^{S_{n}} \\ \mathcal{O}_{3}^{\mathcal{T}}:=((1,2)(3,4),(1,2)(3,5))^{S_{n}} \\ \mathcal{O}_{4}^{\mathcal{T}}:=((1,2)(3,4),(1,3)(2,5))^{S_{n}} \\ \mathcal{O}_{5}^{\mathcal{T}}:=((1,2)(3,4),(1,2)(5,6))^{S_{n}} \\ \mathcal{O}_{6}^{\mathcal{T}}:=((1,2)(3,4),(1,3)(5,6))^{S_{n}} \\ \mathcal{O}_{7}^{\mathcal{T}}:=((1,2)(3,4),(1,5)(2,6))^{S_{n}} \\ \mathcal{O}_{8}^{\mathcal{T}}:=((1,2)(3,4),(1,5)(3,6))^{S_{n}} \\ \mathcal{O}_{9}^{\mathcal{T}}:=((1,2)(3,4),(1,5)(6,7))^{S_{n}} \\ \mathcal{O}_{10}^{\mathcal{T}}:=((1,2)(3,4),(5,6)(7,8))^{S_{n}}\end{array}\right| \begin{aligned} & \mathcal{O}_{3}^{\mathcal{U}}:=(\{1,2,3\},\{1,4,5\})^{S_{n}} \\ & \mathcal{O}_{4}^{\mathcal{U}}:=(\{1,2,3\},\{4,5,6\})^{S_{n}} \\ & \mathcal{O}_{n}\end{aligned}$

TABLE 2. Orbitals of $S_{n}$ on $\mathcal{T}$ and on $\mathcal{U}$

(2) We can choose the $T_{h, i}$ 's in such a way that the images of the vectors of the basis $\mathcal{X}$ under the projection maps $\pi_{h i}^{T}: T \rightarrow T_{h, i}$ are given by the following formula:

$$
x^{\pi_{h i}^{T}}=\sum_{k=1}^{10} q_{h k}^{i i}(\mathcal{T}) \sum_{y \in \Delta_{k}^{\mathcal{T}}(x)} y .
$$

(3) For $h \in\{1, \ldots, 4\}, q_{h k}^{i i}(\mathcal{T})$ is the entry of Table 3 corresponding to the pair $\left(\mathcal{O}_{k}^{\mathcal{T}}, T_{h, i}\right)$.

Proof. The decomposition into irreducible submodules follows from [3, Lemma 6] and the remaining assertions follow from Lemma 2.5 and [3, Tables 8 and 9].

Lemma 4.3. The first eigenmatrix associated to the action of $S_{n}$ on the set $\mathcal{U}$ is displayed in Table 4.

Proof. This follows by routine computation using, e.g., the formulas in [1, Corollary to Theorem 2.9, pp. 219-220].

LEMMA 4.4. Let $U$ be as above, then

(1) U decomposes into irreducible submodules as follows:

$$
U=U_{1} \oplus U_{2} \oplus U_{3} \oplus U_{4},
$$

where $U_{1}$ is the trivial module, $U_{2} \cong S^{(n-1,1)}, U_{3} \cong S^{(n-3,3)}$, and $U_{4} \cong$ $S^{(n-2,2)}$.

(2) We can choose the $U_{i}$ 's in such a way that the images of the vectors of the basis $\mathcal{X}$ under the projection maps $\pi_{i}^{U}: U \rightarrow U_{i}$ are given by the following formula:

$$
x^{\pi_{i}^{U}}=\sum_{k=1}^{4} q_{i k}^{11}(\mathcal{U}) \sum_{y \in \Delta_{k}^{\mathcal{U}}(x)} y .
$$

(3) For $i \in\{1, \ldots, 4\}, q_{i k}^{11}(\mathcal{U})$ is the entry of Table 5 corresponding to the pair $\left(\mathcal{O}_{k}^{\mathcal{U}}, U_{i}\right)$.

Proof. The decomposition into irreducible submodules follows by a standard argument in representation theory of the symmetric group (see [11]). The remaining assertions follow by Lemma 2.5 and Lemma 4.3 . 


\begin{tabular}{|c|c|c|c|c|c|}
\hline & $T_{1,1}$ & $T_{2,1}$ & $T_{3,1}$ & $T_{4,1}$ & $T_{4,2}$ \\
\hline $\mathcal{O}_{1}^{\mathcal{T}}$ & 1 & $n-1$ & $\frac{n(n-1)(n-5)}{6}$ & $\frac{n(n-3)}{2}$ & $\frac{n(n-3)}{2}$ \\
\hline $\mathcal{O}_{2}^{\mathcal{T}}$ & 1 & $n-1$ & $\frac{n(n-1)(n-5)}{6}$ & $\frac{-n(n-3)}{4}$ & $\frac{n(n-3)}{2}$ \\
\hline $\mathcal{O}_{3}^{\mathcal{T}}$ & 1 & $\frac{(3 n-16)(n-1)}{4(n-4)}$ & $\frac{n(n-1)(n-5)(n-10)}{24(n-4)}$ & $\frac{n(n-3)}{4}$ & $\frac{n(n-3)(n-7)}{4(n-4)}$ \\
\hline $\mathcal{O}_{4}^{\mathcal{T}}$ & 1 & $\frac{(3 n-16)(n-1)}{4(n-4)}$ & $\frac{n(n-1)(n-5)(n-10)}{24(n-4)}$ & $\frac{-n(n-3)}{8}$ & $\frac{n(n-3)(n-7)}{4(n-4)}$ \\
\hline $\mathcal{O}_{5}^{\mathcal{T}}$ & 1 & $\frac{(n-1)(n-8)}{2(n-4)}$ & $\frac{-n(n-1)(n-8)}{6(n-4)}$ & $\frac{n(n-3)}{6}$ & $\frac{n(n-3)\left(n^{2}-21 n+92\right)}{12(n-4)(n-5)}$ \\
\hline $\mathcal{O}_{6}^{\mathcal{T}}$ & 1 & $\frac{(n-1)(n-8)}{2(n-4)}$ & $\frac{-n(n-1)(n-8)}{6(n-4)}$ & $\frac{-n(n-3)}{12}$ & $\frac{n(n-3)\left(n^{2}-21 n+92\right)}{12(n-4)(n-5)}$ \\
\hline $\mathcal{O}_{7}^{\mathcal{T}}$ & 1 & $\frac{(n-1)(n-8)}{2(n-4)}$ & $\frac{-n(n-1)(n-8)}{6(n-4)}$ & $\frac{-n(n-3)}{12}$ & $\frac{n(n-3)\left(n^{2}-21 n+92\right)}{12(n-4)(n-5)}$ \\
\hline $\mathcal{O}_{8}^{\mathcal{T}}$ & 1 & $\frac{(n-1)(n-8)}{2(n-4)}$ & $\frac{-n(n-1)(n-8)}{6(n-4)}$ & $\frac{n(n-3)}{24}$ & $\frac{n(n-3)\left(n^{2}-21 n+92\right)}{12(n-4)(n-5)}$ \\
\hline $\mathcal{O}_{9}^{\mathcal{T}}$ & 1 & $\frac{(n-1)(n-16)}{4(n-4)}$ & $\frac{n(n-1)(3 n-22)}{4(n-4)(n-6)}$ & 0 & $\frac{-3 n(n-3)(n-9)}{4(n-4)(n-5)}$ \\
\hline $\mathcal{O}_{10}^{\mathcal{T}}$ & 1 & $\frac{-4(n-1)}{(n-4)}$ & $\frac{-4 n(n-1)}{(n-4)(n-6)}$ & 0 & $\frac{6 n(n-3)}{(n-4)(n-5)}$ \\
\hline
\end{tabular}

TABLE 3. The coefficients $q_{h k}^{i i}(\mathcal{T})$

\begin{tabular}{|c|c|c|c|}
\hline 1 & $3(n-3)$ & $\frac{3}{2}(n-3)(n-4)$ & $\frac{(n-3)(n-4)(n-5)}{6}$ \\
\hline 1 & $2 n-9$ & $\frac{(n-4)(n-9)}{2}$ & $-\frac{1}{2}(n-4)(n-5)$ \\
\hline 1 & $n-7$ & $-2 n+11$ & $n-5$ \\
\hline 1 & -3 & 3 & -1 \\
\hline
\end{tabular}

TABLE 4. The first eigenmatrix $P^{\mathcal{U}}$

\begin{tabular}{|c|c|c|c|c|}
\hline & $U_{1}$ & $U_{2}$ & $U_{3}$ & $U_{4}$ \\
\hline $\mathcal{O}_{1}^{\mathcal{U}}$ & 1 & $n-1$ & $\frac{n(n-3)}{2}$ & $\frac{n(n-1)(n-5)}{6}$ \\
\hline $\mathcal{O}_{2}^{\mathcal{U}}$ & 1 & $\frac{(2 n-9)(n-1)}{3(n-3)}$ & $\frac{n(n-7)}{6}$ & $\frac{-n(n-1)(n-5)}{6(n-3)}$ \\
\hline $\mathcal{O}_{3}^{\mathcal{U}}$ & 1 & $\frac{(n-1)(n-9)}{3(n-3)}$ & $\frac{n(-2 n+11)}{3(n-4)}$ & $\frac{n(n-1)(n-5)}{3(n-3)(n-4)}$ \\
\hline $\mathcal{O}_{4}^{\mathcal{U}}$ & 1 & $\frac{-3(n-1)}{n-3}$ & $\frac{3 n}{n-4}$ & $\frac{-n(n-1)}{(n-3)(n-4)}$ \\
\hline
\end{tabular}

TABLE 5. The second eigenmatrix $Q^{\mathcal{U}}$

Corollary 4.5. A set of representatives for the irreducible $\mathbb{C}\left[S_{n}\right]$-submodules of $V$ is given by

$$
\mathcal{S}:=\left\{1, S^{(n-1,1)}, S^{(n-2,2)}, S^{(n-3,3)}, S^{(n-4,4)}, S^{(n-3,2,1)}, S^{(n-4,2,2)}\right\} .
$$

Moreover

(1) $S^{(n-2,2)}$ has multiplicity 3 in $V$,

(2) the trivial module, $S^{(n-1,1)}$, and $S^{(n-3,3)}$ have multiplicity 2 ,

(3) $S^{(n-4,4)}, S^{(n-3,2,1)}, S^{(n-4,2,2)}$ have multiplicity 1 and appear only as submodules of $T$.

Proof. This follows from Lemma 4.2(1) and Lemma 4.4(1).

4.2. Submodules of Multiplicity 3. In this subsection we assume $S=S^{(n-2,2)}$. We apply the method described in Section 2: let

$$
H_{1} \text { be the stabiliser in } S_{n} \text { of the set }\{1,2,3,4\} \text {, }
$$


and denote the $H_{1}$-orbits of the bitranspositions as follows:

$$
\begin{array}{lll}
\mathcal{P}_{1}:=((1,2)(3,4))^{H_{1}}, & \mathcal{P}_{2}:=((1,2)(3,5))^{H_{1}}, & \mathcal{P}_{3}:=((1,2)(5,6))^{H_{1}}, \\
\mathcal{P}_{4}:=((1,5)(2,6))^{H_{1}}, & \mathcal{P}_{5}:=((1,5)(6,7))^{H_{1}}, & \mathcal{P}_{6}:=((5,6)(7,8))^{H_{1}} .
\end{array}
$$

LEMMA 4.6. The complex permutation module $Z$ of $S_{n}$ on the set of its 4-subsets decomposes into irreducible submodules as follows

$$
Z=1 \oplus S^{(n-1,1)} \oplus S^{(n-2,2)} \oplus S^{(n-3,3)} \oplus S^{(n-4,4)} .
$$

Proof. This follows by standard representation theory of the symmetric groups (see [11]).

Lemma 4.7. $C_{S}\left(H_{1}\right)$ has dimension 1 over $\mathbb{C}$.

Proof. The result follows from Lemma 4.6, since, by Frobenius Reciprocity [6, Lemma 5.2], $\operatorname{dim}_{\mathbb{C}} C_{S}\left(H_{1}\right)$ is equal to the multiplicity of $S$ in the permutation module of $S_{n}$ on the set of 4 -subsets of $\{1, \ldots, n\}$.

Let

$$
u:=\{1,2,3\}+\{1,2,4\}+\{1,3,4\}+\{2,3,4\}
$$

and

$$
s:=\sum_{v \in \mathcal{P}_{3}} v .
$$

Clearly both $u$ and $s$ are $H_{1}$-invariant. We determine the projections $s^{\pi_{41}^{T}}, s^{\pi_{42}^{T}}$, and $u^{\pi_{4}^{U}}$ on $T_{4,1}, T_{4,2}$, and $U_{4}$, respectively, and show that

$$
\left(s^{\pi_{41}^{T}}, s^{\pi_{42}^{T}}, u^{\pi_{4}^{U}}\right)
$$

is the basis $\mathcal{C}_{S}$ for $C_{V\left(S^{(n-2,2)}\right)}\left(H_{1}\right)$ as in Section 2 .

LEMMA 4.8. For $(h, i) \in\{(1,1),(2,1),(3,1),(4,1),(4,2)\}$, we have $s^{\pi_{h i}^{T}} \in C_{T_{h, i}}\left(H_{1}\right)$, and

$$
s^{\pi_{h i}^{T}}=\sum_{l=1}^{6} s_{h i}^{l} \sum_{v \in \mathcal{P}_{l}} v
$$

where

$$
\begin{aligned}
s_{h i}^{1}:= & (n-4)(n-5)\left(q_{h 5}^{i i}(\mathcal{T})+2 q_{h 7}^{i i}(\mathcal{T})\right), \\
s_{h i}^{2}:= & \frac{1}{2}(n-5)\left[2 q_{h 3}^{i i}(\mathcal{T})+4 q_{h 4}^{i i}(\mathcal{T})+(n-6) q_{h 5}^{i i}(\mathcal{T})\right. \\
& \left.+2 q_{h 6}^{i i}(\mathcal{T})+2(n-6) q_{h 7}^{i i}(\mathcal{T})+4 q_{h 8}^{i i}(\mathcal{T})+3(n-6) q_{h 9}^{i i}(\mathcal{T})\right], \\
s_{h i}^{3}:= & q_{1 h}^{i i}(\mathcal{T})+2(n-4) q_{h 3}^{i i}(\mathcal{T})+\frac{(n-6)(n-7)+2}{2} q_{h 5}^{i i}(\mathcal{T})+8(n-6) q_{h 8}^{i i}(\mathcal{T}) \\
& +2(n-6)^{2} q_{h 9}^{i i}(\mathcal{T})+\frac{(n-6)(n-7)}{2} q_{h 10}^{i i}(\mathcal{T}), \\
s_{h i}^{4}:= & q_{h 2}^{i i}(\mathcal{T})+2(n-4) q_{h 4}^{i i}(\mathcal{T})+4(n-6) q_{h 6}^{i i}(\mathcal{T})+\frac{(n-6)(n-7)+2}{2} q_{h 7}^{i i}(\mathcal{T}) \\
& +4(n-6) q_{h 8}^{i i}(\mathcal{T})+2(n-6)^{2} q_{h 9}^{i i}(\mathcal{T})+\frac{(n-6)(n-7)}{2} q_{h 10}^{i i}(\mathcal{T}), \\
s_{h i}^{5}=3 & {\left[\begin{array}{l}
\left.q_{h 3}^{i i}(\mathcal{T})+2 q_{h 4}^{i i}(\mathcal{T})+q_{h 5}^{i i}(\mathcal{T})+(n-7) q_{h 6}^{i i}(\mathcal{T})+2 q_{h 7}^{i i}(\mathcal{T})+2(n-7) q_{h 8}^{i i}(\mathcal{T})\right] \\
\quad+3\left[\frac{(n-7)(n-2)}{2} q_{h 9}^{i i}\left(\mathcal{T}+\frac{(n-7)(n-8)}{2} q_{h 10}^{i i}(\mathcal{T})\right]\right.
\end{array}\right.}
\end{aligned}
$$




$$
s_{h i}^{6}:=6\left[2 q_{h 5}^{i i}(\mathcal{T})+4 q_{h 7}^{i i}(\mathcal{T})+4(n-8) q_{h 9}^{i i}(\mathcal{T})+\frac{(n-8)(n-9)}{2} q_{h 10}^{i i}(\mathcal{T})\right] .
$$

Proof. Since $s \in C_{T}\left(H_{1}\right)$ and $\pi_{h i}^{T}$ is a homomorphism of $\mathbb{C}\left[S_{n}\right]$-modules, $s^{\pi_{h i}^{T}} \in$ $C_{T_{k}}\left(H_{1}\right)$. The formula follows from Lemma 4.4 , since, for $(x, z) \in \mathcal{O}_{k}^{\mathcal{T}}$, the set $\Delta_{k}^{\mathcal{T}}(x)$ is the orbit of $z$ under the action of the stabiliser in $S_{n}$ of $x$.

Denote the $H_{1}$-orbits of $\mathcal{U}$ as follows:

$$
\mathcal{Q}_{1}:=\{1,2,3\}^{H_{1}}, \mathcal{Q}_{2}:=\{1,2,4\}^{H_{1}}, \mathcal{Q}_{3}:=\{1,5,6\}^{H_{1}}, \mathcal{Q}_{4}:=\{5,6,7\}^{H_{1}} .
$$

Lemma 4.9. For $k \in\{1, \ldots, 4\}, u^{\pi_{k}^{U}} \in C_{U_{k}}\left(H_{1}\right)$ and

$$
\begin{aligned}
u^{\pi_{k}^{U}}=\left(q_{k 1}^{11}(\mathcal{U})+3 q_{k 2}^{11}(\mathcal{U})\right) \sum_{v \in \mathcal{Q}_{1}} v+ & \left(2 q_{k 2}^{11}(\mathcal{U})+2 q_{k 3}^{11}(\mathcal{U})\right) \sum_{v \in \mathcal{Q}_{2}} v \\
& +\left(3 q_{k 3}^{11}(\mathcal{U})+q_{k 4}^{11}(\mathcal{U})\right) \sum_{v \in \mathcal{Q}_{3}} v+4 q_{k 4}^{11}(\mathcal{U}) \sum_{v \in \mathcal{Q}_{4}} v
\end{aligned}
$$

Proof. The proof is the same as in the previous lemma.

Corollary 4.10. The multiplicity of $S^{(n-2,2)}$ in $V^{\perp}$ is equal to the corank of the matrix

$$
\Gamma_{4}:=\left(\begin{array}{l}
f\left(s^{\pi_{41}^{T}}, s^{\pi_{41}^{T}}\right) f\left(s^{\pi_{41}^{T}}, s^{\pi_{42}^{T}}\right) f\left(s^{\pi_{41}^{T}}, u^{\pi_{4}^{U}}\right) \\
f\left(s^{\pi_{42}^{T}}, s^{\pi_{41}^{T}}\right) f\left(s^{\pi_{42}^{T}}, s^{\pi_{42}^{T}}\right) f\left(s^{\pi_{42}^{T}}, u^{\pi_{4}^{U}}\right) \\
f\left(u^{\pi_{4}^{U}}, s^{\pi_{41}^{T}}\right) f\left(u^{\pi_{4}^{U}}, s^{\pi_{42}^{T}}\right) f\left(u^{\pi_{4}^{U}}, u^{\pi_{4}^{U}}\right)
\end{array}\right)
$$

Proof. An easy, though tedious, computation shows that none of $s^{\pi_{41}^{T}}, s^{\pi_{42}^{T}}$, and $u^{\pi_{4}^{U}}$ is the zero vector. Therefore, since they belong to distinct irreducible components of $V$, $\left(s^{\pi_{41}^{T}}, s^{\pi_{42}^{T}}, u^{\pi_{4}^{U}}\right)$ is a basis of $C_{V\left(S^{(n-2,2)}\right)}\left(H_{1}\right)$. The result then follows by Corollary 2.4 and Lemma 4.7.

4.3. Submodules of multiplicity 2. In this subsection assume

$$
S \in\left\{1, S^{(n-1,1)}, S^{(n-3,3)}\right\} .
$$

In this case, we still have $\operatorname{dim}_{\mathbb{C}} C_{S}\left(H_{1}\right)=1$, but the elements $s^{\pi_{k 1}^{T}}$ are equal to 0 if $k \in\{1,2,3\}$ and $n=8$. Not to treat separately these cases we replace the subgroup $H_{1}$ and the element $s$ by more suitable ones. Let

$$
t:=(1,2)(3,4) \text {, and } H_{2} \text { be the centraliser in } S_{n} \text { of } t .
$$

Obviously $t$ is $H_{2}$-invariant and, in the same way as in Lemma 4.7 using [3, Lemma 6] in place of Lemma 4.6, one proves that

$$
\operatorname{dim}_{\mathbb{C}} C_{S}\left(H_{2}\right)=1 .
$$

Denote the $H_{2}$-orbits of $\mathcal{T}$ as follows:

$$
\begin{array}{rll}
\mathcal{R}_{1}:=((1,2)(3,4))^{H_{2}}, & \mathcal{R}_{2}:=((1,3)(2,4))^{H_{2}}, & \mathcal{R}_{3}:=((1,2)(3,5))^{H_{2}}, \\
\mathcal{R}_{4}:=((1,3)(2,5))^{H_{2}}, & \mathcal{R}_{5}:=((1,2)(5,6))^{H_{2}}, & \mathcal{R}_{6}:=((1,3)(5,6))^{H_{2}}, \\
\mathcal{R}_{7}:=((1,5)(2,6))^{H_{2}}, & \mathcal{R}_{8}:=((1,5)(3,6))^{H_{2}}, & \mathcal{R}_{9}:=((1,5)(6,7))^{H_{2}}, \\
\mathcal{R}_{10}:=((5,6)(7,8))^{H_{2}} . &
\end{array}
$$

LEMma 4.11. For $k \in\{1, \ldots, 3\}, t^{\pi_{k 1}^{T}} \in C_{T_{k, 1}}\left(H_{2}\right)$, and

$$
t^{\pi_{k, 1}^{T}}=\sum_{h=1}^{10} q_{k h}^{11}(\mathcal{T}) \sum_{v \in \mathcal{R}_{h}} v
$$


Proof. Clearly $t \in C_{T}\left(H_{2}\right)$ and so $t^{\pi_{k 1}^{T}} \in C_{T_{k, 1}}\left(H_{2}\right)$, since $\pi_{k 1}^{T}$ is a homomorphism of $\mathbb{C}\left[S_{n}\right]$-modules. The formula follows from Lemma 4.2 , since, for $(x, z) \in \mathcal{O}_{k}^{\mathcal{T}}, \Delta_{k}^{\mathcal{T}}(x)=$ $z^{C_{S_{n}}(x)}$.

Let $u$ be as in Subsection 4.2. Since $H_{2} \leqslant H_{1}, u$ and its projections $u^{\pi_{1}^{U}}, u^{\pi_{2}^{U}}$, and $u^{\pi_{3}^{U}}$ are $H_{2}$-invariant. As above, for $k \in\{1,2,3\},\left(t^{\pi_{k 1}^{T}}, u^{\pi_{k}^{U}}\right)$ is a basis of $C_{V\left(U_{k}\right)}\left(H_{2}\right)$, whence the following result holds.

Corollary 4.12. For $S \in\left\{1, S^{(n-1,1)}, S^{(n-3,3)}\right\}$, the multiplicity of $S$ in $V^{\perp}$ is equal to the corank of the matrix

$$
\Gamma_{k}:=\left(\begin{array}{l}
f\left(t^{\pi_{k 1}^{T}}, t^{\pi_{k 1}^{T}}\right) f\left(t^{\pi_{k 1}^{T}}, u^{\pi_{k}^{U}}\right) \\
f\left(t^{\pi_{k 1}^{T}}, u^{\pi_{k}^{U}}\right) f\left(u^{\pi_{k}^{U}}, u^{\pi_{k}^{U}}\right)
\end{array}\right)
$$

for $k=1,2,3$ respectively.

4.4. Submodules of multiplicity 1. Finally, assume

$$
S \in\left\{S^{(n-4,4)}, S^{(n-3,2,1)}, S^{(n-4,2,2)}\right\} .
$$

By Corollary 4.5 and Corollary 2.2, we may restrict to the action of $S_{n}$ on $\mathcal{T}$ and apply the results in [3].

LEMMA 4.13.

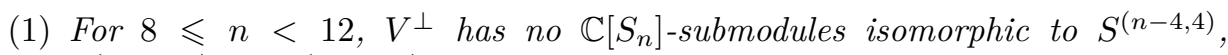
$S^{(n-3,2,1)}$, or $S^{(n-4,2,2)}$,

(2) for $n=12, V^{\perp}$ has no $\mathbb{C}\left[S_{n}\right]$-submodules isomorphic to $S^{(n-4,4)}$ or $S^{(n-4,2,2)}$, and has exactly one $\mathbb{C}\left[S_{n}\right]$-submodule isomorphic to $S^{(n-3,2,1)}$.

Proof. Let $S \in\left\{S^{(n-4,4)}, S^{(n-3,2,1)}, S^{(n-4,2,2)}\right\}$. By Corollary 2.2, the multiplicity of $S$ in $V^{\perp}$ is equal to the multiplicity of $S$ in $T \cap T^{\perp}$. The result then follows from [3, Table 13].

4.5. The MAtrices $\Gamma_{k}$. In this subsection we show how to compute the entries of the matrices $\Gamma_{1}, \ldots, \Gamma_{4}$. Note first that the values $f\left(t^{\pi_{k i}^{T}}, t^{\pi_{k i}^{T}}\right)$ and $f\left(s^{\pi_{k i}^{T}}, s^{\pi_{k i}^{T}}\right)$ (resp. $f\left(u^{\pi_{k}^{U}}, u^{\pi_{k}^{U}}\right)$ ) can be computed inside the module $T$, (resp. $\left.U\right)$, so we can avoid a direct computation by applying the results from [3] (resp. Table 6).

LEMMA 4.14. Let $\kappa: V \times V \rightarrow \mathbb{C}$ be the hermitian form on $V$ with respect to which $\mathcal{X}$ is an orthonormal basis. Then, for every $v \in T_{k, i}$ and $w \in U_{l}$, where $(k, i) \in$ $\{(1,1),(2,1),(3,1),(4,1),(4,2)\}$ and $l \in\{1, \ldots, 4\}$, we have

$$
f(v, v)=\kappa(v, v) \delta_{k i} \text { and } f(w, w)=\kappa(w, w) \epsilon_{l},
$$

where the $\delta_{k i}$ 's and the $\epsilon_{l}$ 's are listed in Table 6 .

\begin{tabular}{|c|c|c|c|}
\hline$k$ & $\delta_{k 1}$ & $\delta_{k 2}$ & $\epsilon_{l}$ \\
\hline 1 & $\frac{5}{128} n^{3}-\frac{25}{128} n^{2}-\frac{15}{64} n+\frac{45}{16}$ & & $\frac{8}{135} n^{2}+\frac{16}{27} n-\frac{32}{45}$ \\
\hline 2 & $\frac{5}{512} n^{3}-\frac{35}{512} n^{2}-\frac{15}{64} n+\frac{45}{16}$ & & $\frac{8}{405} n^{2}+\frac{56}{135} n-\frac{32}{45}$ \\
\hline 3 & $\frac{15}{128}(18-n)$ & & $\frac{752}{675}$ \\
\hline 4 & $\frac{5}{768}(n-32)(n-13)$ & $\frac{37}{768} n^{2}-\frac{97}{256} n+\frac{311}{192}$ & $\frac{8}{2025}(31 n+158)$ \\
\hline
\end{tabular}

TABLE 6. Values of $\delta_{k i}$ and $\epsilon_{l}$ 
Proof. Since $T_{k, i}\left(\operatorname{resp} U_{k}\right)$ is an irreducible $\mathbb{C}\left[S_{n}\right]$-module, by [12, p. 534], any two $S_{n}$-invariant hermitian forms on $T_{k}$ (resp. $U_{k}$ ) differ only by a scalar. The result for $T_{k}$ then follows from [3, Table 13], of which Table 6 is an extract. The same argument gives the values $\epsilon_{k}$ from the first eigenmatrix associated to the action of $S_{n}$ on $U$ (Table 4).

The remaining entries of the matrices $\Gamma_{i}$ have been computed, using the formulae in Section A.

\begin{tabular}{|c|c|}
\hline$k$ & $f\left(u^{\pi_{k}^{U}}, u^{\pi_{k}^{U}}\right)$ \\
\hline 1 & $\frac{64}{405} n(n-1)(n-2)\left(n^{2}+10 n-12\right)$ \\
\hline 2 & $\frac{16}{405} \frac{n(n-1)^{2}(n-2)(n-4)\left(n^{2}+21-36\right)}{(n-3)}$ \\
\hline 3 & $\frac{64}{405} \frac{n^{2}(n-1)^{2}(n-2)(n-5)(n-6)}{(n-3)}$ \\
\hline 4 & $\frac{208}{1215} n^{2}(n-1)(n-2)(n-5)\left(n-\frac{16}{13}\right)$ \\
\hline
\end{tabular}

TABLE 7. Values of $f\left(u^{\pi_{k}^{U}}, u^{\pi_{k}^{U}}\right)$

\begin{tabular}{|c|c|}
\hline$i$ & $\operatorname{det}\left(\Gamma_{i}\right)$ \\
\hline 1 & $-\frac{1}{108} n^{2}(n-1)^{2}(n-2)^{2}(n-3)(n-12)\left(n^{2}-2 n+6\right)$ \\
\hline 2 & $-\frac{1}{288} n^{2}(n-1)^{4}(n-2)^{2}(n-4)(n-12)\left(n^{2}-3 n+12\right)$ \\
\hline 3 & $-\frac{1}{2592} n^{4}(n-1)^{4}(n-2)^{2}(n-5)^{2}(n-6)(n-12)$ \\
\hline 4 & $-\frac{1}{2^{21 \cdot 32}} n^{6}(n-1)^{4}(n-2)^{4}(n-3)^{4}(n-4)(n-5)^{2}(n-7)^{2}$ \\
& $(n-11)(n-12)^{2}(n-14)^{2}\left(n^{2}-3 n+12\right)$ \\
\hline
\end{tabular}

TABLE 8. Determinants of matrices $\Gamma_{i}$

4.6. Proof of Theorem 1.1. The first assertion follows from Lemma 4.1. Assume

$$
S \in\left\{S^{(n-4,4)}, S^{(n-4,2,2)}, S^{(n-3,2,1)}\right\},
$$

then the multiplicities of $S$ in $V^{\perp}$ are given in Lemma 4.13. Assume

$$
S \in\left\{1, S^{(n-1,1)}, S^{(n-3,3)}\right\} .
$$

By Tables 7 and 8 , for $i \in\{1,2,3\}$, the corank of $\Gamma_{i}$ is 0 , if $n \neq 12$, and 1 , if $n=12$. The result then follows from Corollary 4.12. Finally assume

$$
S=S^{(n-2,2)} \text {. }
$$

If $n \leqslant 10$, by Table $8, \operatorname{det}\left(\Gamma_{4}\right) \neq 0$, so the result follows from Corollary 4.10 . If $n=11$, then $\operatorname{det}\left(\Gamma_{4}\right)=0$ and, since, by Lemma $4.1, T \cap T^{\perp}=\{0\}$, it follows that $\Gamma_{4}$ has rank 2. The result then follows from Corollary 4.10 . If $n=12, \operatorname{det}\left(\Gamma_{4}\right)=0$, hence, $\Gamma_{4}$ has rank at most 2 . Let $\beta, \delta$ be the submatrices of $\Gamma_{4}$ defined as follows

$$
\beta:=\left(\begin{array}{l}
f\left(s^{\pi_{5}^{V}}, s^{\pi_{5}^{V}}\right) f\left(s^{\pi_{5}^{V}}, u^{\pi_{4}^{U}}\right) \\
f\left(u^{\pi_{4}^{U}}, s^{\pi_{5}^{V}}\right) f\left(u^{\pi_{4}^{U}}, u^{\pi_{4}^{U}}\right)
\end{array}\right), \quad \delta:=\left(\begin{array}{l}
f\left(s^{\pi_{4}^{V}}, s^{\pi_{4}^{V}}\right) f\left(s^{\pi_{4}^{V}}, s^{\pi_{5}^{V}}\right) \\
f\left(s^{\pi_{5}^{V}}, s^{\pi_{4}^{V}}\right) f\left(s^{\pi_{5}^{V}}, s^{\pi_{5}^{V}}\right)
\end{array}\right)
$$

Using the formulae in Section A, we get that the determinant of $\beta$ is equal to

$$
-\frac{1}{2^{9} \cdot 3^{4} \cdot 5} n^{4}(n-1)^{3}(n-2)^{3}(n-3)^{2}(n-4)(n-5)^{2}(n-12)\left(n^{2}+117 n-148\right)
$$


that vanishes when $n=12$. By Lemma 4.14 and [3, Table 13 and Equation 25], the determinant of $\delta$ is a multiple of

$$
\frac{15}{2^{16}}(n-12)\left(n^{3}-56 n^{2}+411 n-1596\right)
$$

that also vanishes when $n=12$. Since the maximum rank of the $2 \times 2$ submatrices of $\Gamma_{4}$ is equal to the maximum rank of the two matrices $\beta$ and $\delta$, we get that $\Gamma_{4}$ has rank 1, so, again, the claim follows from Corollary 4.10 .

\section{Appendix A.}

We give here some formulae we used to compute the entries of matrices $\Gamma_{i}$. They have been obtained by straightforward computation using Lemma 1 and the following easy observation.

Lemma A.1. Let $L \leqslant S_{n}$ and let $\mathcal{O}_{1}$ and $\mathcal{O}_{2}$ be two L-orbits on $\mathcal{X}$. Then, for every $x \in \mathcal{O}_{1}$, we have

$$
f\left(\sum_{v \in \mathcal{O}_{1}} v, \sum_{w \in \mathcal{O}_{2}} w\right)=\left|\mathcal{O}_{1}\right| \sum_{y \in \mathcal{O}_{2}} f(x, y) .
$$

Let $a, b \in C_{T}\left(H_{2}\right), d \in C_{T}\left(H_{1}\right)$, and $c \in C_{U}\left(H_{2}\right)$ and suppose that

$$
a=\sum_{h=1}^{6} a_{h} \sum_{v \in \mathcal{P}_{h}} v, \quad b=\sum_{h=1}^{6} b_{h} \sum_{v \in \mathcal{P}_{h}} v, c=\sum_{h=1}^{4} c_{h} \sum_{v \in \mathcal{Q}_{h}} v, d=\sum_{h=1}^{10} d_{h} \sum_{v \in \mathcal{R}_{h}} v
$$

with $a_{i}, b_{i}, c_{i}, d_{i} \in \mathbb{C}$. Then

$$
\begin{aligned}
f(a, b)= & a_{1} b_{1} \frac{15}{4}+\left(a_{1} b_{2}+a_{2} b_{1}\right) \frac{75}{64}(n-4)+\left(a_{1} b_{3}+a_{3} b_{1}\right) \frac{15}{32}(n-4)(n-5) \\
& +\left(a_{1} b_{4}+a_{4} b_{1}\right) \frac{45}{64}(n-4)(n-5)+\left(a_{1} b_{5}+a_{5} b_{1}\right) \frac{15}{128}(n-4)(n-5)(n-6) \\
& +\left(a_{1} b_{6}+a_{6} b_{1}\right) 0+a_{2} b_{2} \frac{15}{64}(n-4)(25 n-46) \\
& +\left(a_{2} b_{3}+a_{3} b_{2}\right) 3 \frac{55}{128}(n-4)^{2}(n-5) \\
& +\left(a_{2} b_{4}+a_{4} b_{2}\right) \frac{45}{64}(n-4)(n-5)(3 n-10) \\
& +\left(a_{2} b_{5}+a_{5} b_{2}\right) \frac{15}{128}(n-4)(n-5)(n-6)(3 n+2) \\
& +\left(a_{2} b_{6}+a_{6} b_{2}\right) \frac{15}{128}(n-4)(n-5)(n-6)(n-7) \\
& +3 a_{3} b_{3}(n-4)(n-5)\left(\frac{13}{128} n^{2}-\frac{99}{128} n+\frac{37}{16}\right) \\
& +\left(a_{3} b_{4}+a_{4} b_{3}\right) \frac{9}{64}(n-4)(n-5)\left(2 n^{2}-11 n+4\right) \\
& +\left(a_{3} b_{5}+a_{5} b_{3}\right) \frac{15}{256}(n-4)(n-5)(n-6)\left(n^{2}+3 n-44\right) \\
& +\left(a_{3} b_{6}+a_{6} b_{3}\right) \frac{15}{256}(n-4)^{2}(n-5)(n-6)(n-7) \\
& +a_{4} b_{4} \frac{9}{128}(n-4)(n-5)\left(11 n^{2}-53 n+52\right) \\
& +\left(a_{4} b_{5}+a_{5} b_{4}\right) \frac{15}{128}(n-4)(n-5)(n-6)\left(n^{2}+5 n-62\right) \\
& \left.+a_{4} b_{6}+a_{6} b_{4}\right) \frac{15}{128}(n-4)(n-5)^{2}(n-6)(n-7) \\
& (n-5) \\
& (n-5) \\
&
\end{aligned}
$$




$$
\begin{aligned}
& +a_{5} b_{5} \frac{1}{256}(n-4)(n-5)(n-6)\left(5 n^{3}+115 n^{2}-1820 n+6180\right) \\
& +\left(a_{5} b_{6}+a_{6} b_{5}\right) \frac{5}{256}(n-4)(n-5)(n-6)(n-7)\left(3 n^{2}-31 n+66\right) \\
& +a_{6} b_{6} \frac{(n-4)(n-5)(n-6)(n-7)}{8}\left(\frac{5}{128} n^{3}-\frac{85}{128} n^{2}+\frac{205}{64} n-\frac{15}{8}\right) \text {, } \\
& f(a, c)=\left(c_{1}+3 c_{2}\right)\left[a_{1} \frac{4}{3}+a_{2}(n-4) \frac{17}{3}+a_{3}(n-4)(n-5) \frac{5}{3}\right. \\
& \left.+a_{4}(n-4)(n-5)+a_{5}(n-4)(n-5)(n-6) \frac{1}{6}\right] \\
& +2\left(c_{2}+c_{3}\right)\left[a_{1}(n-4) \frac{13}{6}+a_{2}(n-4) \frac{(16 n-55)}{3}\right. \\
& +a_{3}(n-4)^{2}(n-5) \frac{13}{12}+a_{4}(n-4)(n-5)(n+3) \\
& +a_{5}(n-4)(n-5)(n-6)(n+9) \frac{1}{6} \\
& \left.+a_{6}(n-4)(n-5)(n-6)(n-7) \frac{1}{12}\right] \\
& +\left(3 c_{3}+c_{4}\right)\left[a_{1}(n-4)(n-5) \frac{1}{6}+a_{2}(n-4)(n-5)(3 n+10) \frac{1}{6}\right. \\
& +a_{3}(n-4)(n-5)\left(\frac{13}{6}+(n-6)+(n-6)(n-7) \frac{1}{12}\right) \\
& +a_{4}(n-4)(n-5)\left(2+(n-6) \frac{13}{3}+(n-6)(n-7) \frac{1}{6}\right) \\
& +a_{5}(n-4)(n-5)(n-6)\left(\frac{17}{6}+\frac{11}{9}(n-7)\right. \\
& \left.+\frac{1}{36}(n-7)(n-8)\right) \\
& \left.+a_{6}(n-4)(n-5)(n-6)(n-7)\left(\frac{13}{36}+\frac{1}{18}(n-8)\right)\right] \\
& +4 c_{4}\left[a_{2}(n-4)(n-5)(n-6) \frac{1}{6}\right. \\
& +a_{3}(n-4)(n-5)(n-6)\left(\frac{1}{12}(n-7)+\frac{3}{4}\right) \\
& +a_{4}(n-4)(n-5)(n-6)\left(\frac{1}{6}(n-7)+\frac{1}{3}\right) \\
& +a_{5}(n-4)(n-5)(n-6)\left(\frac{2}{9}+\frac{13}{18}(n-7)\right. \\
& \left.+\frac{1}{12}(n-8)(n-7)\right) \\
& +a_{6} \frac{1}{8}(n-4)(n-5)(n-6)(n-7)\left(\frac{4}{9}+\frac{13}{18}(n-8)\right. \\
& \left.\left.+\frac{1}{18}(n-8)(n-9)\right)\right] \text {, }
\end{aligned}
$$


and

$$
\begin{aligned}
f(d, c)=\left(c_{1}+3 c_{2}\right)\left[\frac{4}{9}\right. & d_{1}+\frac{8}{9} d_{2}+4(n-4) \frac{17}{36} d_{3}+4(n-4) \frac{17}{18} d_{4} \\
& +2(n-4)(n-5) \frac{5}{18} d_{5}+4(n-4)(n-5) \frac{5}{18} d_{6} \\
& +\frac{(n-4)(n-5)}{3} d_{7}+2 \frac{(n-4)(n-5)}{3} d_{8} \\
& \left.\left.+\frac{(n-4)(n-5)(n-6)}{6} d_{9}\right)\right] \\
+2\left(c_{2}+c_{3}\right)[ & \frac{13}{18}\left(d_{1}+2 d_{2}\right)(n-4)+d_{3}(n-4)\left(\frac{16}{9} n-\frac{55}{9}\right) \\
& +2 d_{4}(n-4)\left(\frac{16}{9} n-\frac{55}{9}\right)+d_{5}(n-4)(n-5) \frac{13}{36}(n-4) \\
& +d_{6}(n-4) \frac{13}{18}(n-5)(n-4) \\
& +d_{7}(n-4)(n-5)(n+3) \frac{1}{3}+d_{8}(n-4)(n-5)(n+3) \frac{2}{3} \\
& +d_{9}(n-4)(n-5)(n-6)(n+9) \frac{1}{6} \\
& \left.+d_{10}(n-4)(n-5)(n-6)(n-7) \frac{1}{12}\right] \\
4 c_{4}\left\{d_{3}(n-4)\right. & (n-5)(n-6) \frac{1}{18}+d_{4}(n-4)(n-5)(n-6) \frac{1}{9} \\
& +d_{5}(n-4)(n-5)(n-6)\left[\frac{1}{36}(n-7)+\frac{1}{4}\right] \\
& \left.+d_{10}(n-4)(n-5)(n-6)(n-7)\left[\frac{13}{36}+(n-8) \frac{1}{18}\right]\right\} \\
+\left(3 c_{3}+c_{4}\right)\{ & d_{1}(n-4)(n-5) \frac{1}{18}+d_{2}(n-4)(n-5) \frac{1}{9} \\
& +d_{3}(n-4)(n-5)(3 n+10) \frac{1}{18} \\
& +d_{4}(n-4)(n-5)(3 n+10) \frac{1}{9} \\
& +d_{5}(n-4)(n-5)\left[\frac{13}{18}+(n-6) \frac{1}{3}+(n-6)(n-7) \frac{1}{36}\right] \\
& +d_{6}(n-4)(n-5)\left[\frac{13}{9}+\frac{2}{3}(n-6)+(n-6)(n-7) \frac{1}{18}\right] \\
& +d_{7}(n-4)(n-5)\left[\frac{2}{3}+\frac{13}{9}(n-6)+(n-6)(n-7) \frac{1}{18}\right] \\
& +d_{8}(n-4)(n-5)\left[\frac{2}{3}+\frac{13}{9}(n-6)+(n-6)(n-7) \frac{1}{18}\right] \\
& \\
& \\
&
\end{aligned}
$$




$$
\begin{aligned}
& +d_{6}(n-4)(n-5)(n-6)\left[\frac{1}{18}(n-7)+\frac{1}{2}\right] \\
& +d_{7}(n-4)(n-5)(n-6)\left[\frac{1}{18}(n-7)+\frac{1}{9}\right] \\
& +d_{8}(n-4)(n-5)(n-6)\left[\frac{1}{9}(n-7)+\frac{2}{9}\right] \\
& +d_{9}(n-4)(n-5)(n-6)\left[\frac{2}{9}+\frac{13}{18}(n-7)+\frac{1}{12}(n-8)(n-7)\right] \\
& +d_{10} \frac{(n-4)(n-5)(n-6)(n-7)}{8}\left[\frac{4}{9}+\frac{13}{18}(n-8)\right. \\
& \left.\left.+\frac{1}{18}(n-8)(n-9)\right]\right\} .
\end{aligned}
$$

\section{REFERENCES}

[1] Eiichi Bannai and Tatsuro Ito, Algebraic combinatorics. I: Association schemes, Mathematics Lecture Note Series, The Benjamin/Cummings Publishing Company, 1984.

[2] Alonso Castillo-Ramirez and Alexander A. Ivanov, The axes of a Majorana representation of $A_{12}$, in Groups of exceptional type, Coxeter groups and related geometries (Bangalore, 2012), Springer Proceedings in Mathematics \& Statistics, vol. 82, Springer, 2014, pp. 159-188.

[3] Clara Franchi, Alexander A. Ivanov, and Mario Mainardis, Standard Majorana representations of the symmetric groups, J. Algebr. Comb. 44 (2016), no. 2, 265-292.

[4] , The 2A-Majorana representations of the Harada-Norton group, Ars Math. Contemp. 11 (2016), no. 1, 175-187.

[5] Donald G. Higman, Coherent configurations. I: Ordinary representation theory, Geom. Dedicata 4 (1975), 1-32.

[6] I. Martin Isaacs, Character theory of finite groups, Dover Publications, 1994.

[7] Alexander A. Ivanov, The Monster group and Majorana involutions, Cambridge Tracts in Mathematics, vol. 176, Cambridge University Press, 2009.

[8] $\ldots$, On Majorana representations of $A_{6}$ and $A_{7}$, Commun. Math. Phys. 307 (2011), no. 1, $1-16$.

[9] Alexander A. Ivanov, Dmitrii V. Pasechnik, Ákos Seress, and Sergey V. Shpectorov, Majorana representations of the symmetric group of degree 4, J. Algebra 324 (2010), no. 9, 2432-2463.

[10] Alexander A. Ivanov and Ákos Seress, Majorana representations of $A_{5}$, Math. Z. 272 (2012), no. 1-2, 269-295.

[11] Gordon D. James, The representation theory of the symmetric groups, vol. 682, Springer, 1978.

[12] Serge Lang, Algebra, Graduate Texts in Mathematics, vol. 211, Springer, 2002.

[13] Simon P. Norton, F and other simple groups, Ph.D. thesis, University of Cambridge (UK), 1975.

[14] _ The Monster algebra: Some new formulae, in Moonshine, the monster, and related topics. Joint summer research conference on moonshine, the monster, and related topics (Mount Holyoke College, 1994), Contemporary Mathematics, vol. 193, American Mathematical Society, 1996, pp. 297-306.

[15] Jean-Pierre Serre, Linear representations of finite groups, Graduate Texts in Mathematics, vol. 42, Springer, 1977.

Clara Franchi, Dipartimento di Matematica e Fisica, Università Cattolica del Sacro Cuore, Via Musei 41, I-25121 Brescia, Italy

E-mail : clara.franchi@unicatt.it

Alexander A. Ivanov, Department of Mathematics, Imperial College, 180 Qeen's Gt., London, SW7 2AZ, UK

E-mail : a.ivanov@imperial.ac.uk

Mario Mainardis, Dipartimento di Scienze Matematiche, Informatiche e Fisiche, Università degli Studi di Udine, via delle Scienze 206, I-33100 Udine, Italy

E-mail : mario.mainardis@uniud.it 